\title{
Waiver of Authorization for Release of Confidential Health Information
}

National Cancer Institute

\section{Source}

National Cancer Institute. Waiver of Authorization for Release of Confidential Health

Information. NCI Thesaurus. Code C70687.

A waiver of the requirement for authorization for use or disclosure of private health information. The waiver of authorization for release of confidential health information may be obtained from the IRB by a researcher. A waiver of authorization can be approved only under limited circumstances defined by HIPAA if specific criteria have been met. 